\title{
ESTUDIO DE TURBAS Y RESIDUOS AVÍCOLAS PROCEDENTES DE POLLO DE ENGORDE COMO COMPONENTE DE SUSTRATOS DE CULTIVO
}

\author{
María del Mar DELGADO ARROYO ${ }^{*}$, Rosario MIRALLES DE IMPERIAL HORNEDO ${ }^{1}$, \\ Alberto MASAGUER RODRÍGUEZ ${ }^{2}$ y José Valero MARTÍN SÁNCHEZ ${ }^{1}$
}

\footnotetext{
${ }^{1}$ Departamento de Medio Ambiente, Instituto Nacional de Investigación y Tecnología Agraria y Alimentaria (INIA). Carretera de La Coruña, kilómetro 7.5, Madrid, España, C.P. 28040

${ }^{2}$ Departamento de Edafología, Escuela Técnica Superior de Ingeniería (ETSI) Agronómica, Alimentaria y de Biosistemas, Universidad Politécnica de Madrid (UPM). Ciudad Universitaria, Madrid, España, C.P. 28040

*Autor para correspondencia: delgado@inia.es
}

(Recibido febrero 2016; aceptado febrero 2016)

Palabras clave: turba rubia, turba negra, pollinaza, propiedades físicas e hidrofísicas

\section{RESUMEN}

\begin{abstract}
En este trabajo se aborda el estudio del aprovechamiento de los residuos avícolas procedentes de la cría de pollo de engorde sobre diferentes tipos de material de cama (paja o serrín) y las mezclas de residuo con dos sustratos comerciales con $100 \%$ de turba (turba negra y turba rubia). Lo anterior con el fin de formular sustratos para la producción de plantas ornamentales que reduzcan la utilización de turba como sustrato principal. Las caracterizaciones físicas e hidrofísicas se realizaron sobre dos tipos de materiales: residuo procedente de cría de pollo de engorde sobre paja y pollo de engorde sobre serrín. Estos dos residuos fueron mezclados cada uno con turba negra y turbia rubia al 0 y $10 \%(\mathrm{v} / \mathrm{v})$. Los resultados obtenidos de las determinaciones físicas e hidrofísicas de los sustratos estudiados muestran baja densidad aparente y elevada porosidad, lo que da lugar a un posible exceso en la retención de agua. De la curva de humedad a bajas succiones se interpreta que las mezclas presentan buena capacidad de aireación superior a 20, excepto el sustrato obtenido de la mezcla 90 turba negra +10 pollo de engorde sobre serrín. Por otra parte el agua fácilmente utilizable y la capacidad compensadora de agua es deficiente en agua y el agua difícilmente utilizable es muy elevada. Finalmente, el parámetro R, que se define como la altura en $\mathrm{cm}$ de la columna de agua en la que se igualan los volúmenes de agua y de aire, refleja que estos sustratos tienen suficiente.
\end{abstract}

Key words: black peat, Sphagnum peat, broiler manure, physical and hydrophysical properties

\begin{abstract}
This work studied the use of waste from the breeding of broiler on different types of bedding material (straw or sawdust) and its mixture with two commercial peat substrates (black peat and Sphagnum peat). The aim of this study was to achieve the formulation of substrates for the production of ornamental plants, which would reduce the use of peat as the major substrate. Physical and hydrophysical characterization were carried out on two types of materials: manure from the breeding of broiler on straw and broiler on sawdust.
\end{abstract}


Each material was mixed with black peat and Sphagnum peat at 0 and $10 \%(\mathrm{v} / \mathrm{v})$. In relation to the physical and hydrophysical properties of the substrates studied, results showed a low bulk density, a high porosity and excessive water retention. From the curve humidity to low suctions, it was found that the mixtures had a good aeration capacity. This aeration capacity of all of the mixtures was greater than 20, except for the substrate obtained by mixing 90 black peat and 10 broiler sawdust. Moreover the water easily usable and the water buffering capacity is poor in water and hardly usable water is very high. Finally, the $\mathrm{R}$ parameter ( $\mathrm{cm}$ of water column height to which the parameter volumes of water and air are equal), show that these substrates have sufficient water easily usable.

\section{INTRODUCCIÓN}

España es uno de los mayores productores europeos de carne de ave dentro de la Unión Europea (UE). La producción española de pollo de engorde fue de 1165 toneladas en el 2011, lo que supuso un $85.4 \%$ del total de carne de ave producida y un $11.3 \%$ del total de la UE, en cuyo conjunto ocupó el segundo lugar, detrás del Reino Unido. A nivel global, la UE se situó en los últimos años, con una producción de 9.4 millones de $t$, como cuarto productor mundial de carne de pollo por detrás de Estados Unidos (16.7 millones), China (13.2 millones) y Brasil (13 millones). Este sector de la avicultura de carne genera también subproductos, como son las gallinazas de pollos de engorde que hay que tratar de una manera adecuada. Estas gallinazas son el resultado de la mezcla de heces y las diferentes camas utilizadas, como la paja de cereales o el serrín (Delgado et al. 2007, García et al. 2007).

Por otra parte, la turba es un sustrato orgánico de origen natural, nombre genérico que se aplica a diversos materiales que proceden de la descomposición de los vegetales, dependiendo de las condiciones climáticas predominantes durante su formación. Por su, importancia ecológica, la flora específica que los coloniza, la fauna que los habita y por su papel en la regulación de los sistemas hidrológicos de las áreas en las que se ubican, es conveniente destacar la necesidad de protección y conservación para el futuro de estos ecosistemas conocidos como turberas (Schellekens et al. 2011).

La turba de la especie Sphagnum sp. (turba denominada genéricamente rubia) ha sido, durante muchos años, el componente más importante y ampliamente utilizado en los medios de cultivo de las plantas ornamentales en contenedor. La idoneidad de la turba rubia es consecuencia de sus características intrínsecas, destacando sus excelentes propiedades físicas (densidad aparente, porosidad y capacidad de retención de agua). La turba denominada negra es de color oscuro, está muy descompuesta y procede de capas más profundas del suelo.

España es un importante consumidor de sustratos para la producción hortícola (semilleros fundamentalmente) y ornamental. Al no ser un país productor de turba, depende de terceros países y de las condiciones climáticas en las épocas de extracción de las mismas. España importa $99.2 \%$ de la turba que consume, lo que representa un gasto anual de más de 29 millones de euros (IGME 2010). Estos condicionantes relativos a la extracción y al comercio de turba influyen directamente en el precio de los sustratos, con incrementos anuales de entorno al $6 \%$. Todo ello justifica las investigaciones realizadas para la sustitución de la turba por otros materiales.

Utilizar sustratos que no empleen turbas, recursos no renovables o que reduzcan su uso, sustituyéndolas en parte por residuos ganaderos como son las gallinazas de la industria avícola, puede contribuir a reciclar estos residuos y aprovechar su contenido en nutrientes. Sin embargo, su utilización requiere buscar la proporción correcta de estos residuos en las mezclas para el cultivo de plantas en maceta, con el fin de obtener mayor calidad de la planta y un reciclado máximo del residuo, minimizando los posibles efectos negativos que pueda tener sobre el cultivo.

Debido a que la industria hortícola es uno de los principales consumidores de subproductos orgánicos para ser utilizados como medio de cultivo (López et al. 2006), en el Instituto Nacional de Investigaciones Agrarias (INIA) de España, se planteó un ensayo con crisantemo en maceta para investigar el uso de estas gallinazas como sustituto de las turbas en los sustratos de cultivo y ajustar su proporción óptima.

Miralles de Imperial et al. (2008) y Alonso et al. (2012) evaluaron la respuesta del crisantemo a las mezclas de sustratos de turba negra (TN) y turba rubia (TR) con dos tipos de residuos de pollos de engorde (pollinaza), una mezclada con paja de cebada (PEP) y otra con serrín de madera (PES). Los autores encontraron que se puede adicionar 
pollinaza de con cama de serrín de madera en el cultivo de crisantemo en maceta, ya que los mejores resultados se obtuvieron con esta mezcla. La mejora del cultivo por la aplicación del $10 \%$ fue sobre todo evidente cuando se utilizó turba rubia. Con sustrato comercial los resultados fueron muy buenos pero la adición no supuso una mejora notoria en el cultivo respecto al testigo con $100 \%$ de sustrato comercial (mezcla de turba negra y nutriente). Este ensayo también evidenció que la adición de $50 \%$ de pollinaza produce rápidamente la muerte de la planta y el $25 \%$ tampoco lo tolera bien.

El objetivo principal de este trabajo fue la caracterización física e hidrofísica de la turba negra, turba rubia, pollo de engorde-paja y pollo de engorde-serrín para obtener la formulación de los sustratos a ser empleados en la producción de plantas ornamentales.

\section{MATERIALES Y MÉTODOS}

Los ensayos se llevaron a cabo en el Instituto Nacional de Investigaciones Agrarias (INIA) en colaboración con la Universidad Politécnica de Madrid (UPM), España.

Los sustratos utilizados fueron:

- Sustrato comercial o turba negra (TN): Compo Sana ${ }^{\circledR}$ Universal, compuesto por mezcla de turbas (rubia y negra), abono NPK, perlita, Agrosil ${ }^{\circledR}$ (estimulador de raíces) y cal

- Turba rubia 100 \% (TR): Compo Gärtnertof, compuesto por turba ácida de Sphagnum.

Las pollinazas no compostadas (PE) usadas en el ensayo provinieron de dos granjas situadas en la Comunidad de Castilla y León. Están constituidas por mezcla de heces, plumas y cama de paja de cebada, por lo que se le denominó "pollo de engorde-paja"
(PEP) o de serrín de madera "pollo de engorde-serrín" (PES) (García et al. 2007). Las aves destinadas a producción de carne (pollos de engorde) se crían durante un ciclo de aproximadamente 45 días sobre un lecho o cama. Transcurrido este tiempo se retira de la granja dicho lecho para posteriormente preparar las distintas mezclas con turba negra y turba rubia introduciéndolas en un homogeneizador. Las muestras ensayadas aparecen en el cuadro I, en el que se puede observar el porcentaje en volumen (V/V) de la composición de las pollinazas y las turbas empleadas para la formación de los sustratos, con tres repeticiones.

En el cuadro II aparecen las características químicas de las pollinazas de paja y serrín, así como los sustratos de turba negra y de turba rubia utilizados en el ensayo de trabajos anteriores (Delgado et al. 2010). En el cuadro III se presentan los valores de $\mathrm{pH}$ y de conductividad eléctrica (CE) de los sustratos utilizados y sus mezclas. El pH se midió en una suspensión sustrato/agua (1:2.5) con un electrodo de vidrio de un pH-metro Crison micro pH 2001 con compensación automática de temperatura. Para la medida de la $\mathrm{CE}$ $(\mathrm{dS} / \mathrm{m})$, se utilizó un conductímetro Crison micro CM 2201 en un extracto acuoso (1:5.0) (MAPA 1994).

Para la caracterización física e hidrofísica de los sustratos es necesario determinar la curva de retención de humedad (o de liberación de agua) de los materiales que se utilizan como sustratos de cultivo. Esta curva fue desarrollada por De Boodt et al. (1974) y representa el porcentaje de humedad en volumen entre el porcentaje de materia seca en volumen, frente a la tensión aplicada a los sustratos en $\mathrm{cm}$ de altura de la columna de agua $(10,50$ y $100 \mathrm{~cm})$.

El ensayo fue realizado utilizando un dispositivo de lecho de arena, según AENOR (2012) y los parámetros estudiados fueron:

- Humedad en peso $(\mathrm{H})$ : se define como la masa de agua (en g) que hay en $100 \mathrm{~g}$ de materia seca. Desecada en estufa a $105^{\circ} \mathrm{C}(\%)$.

CUADRO I. COMPOSICIÓN DE LAS POLLINAZAS Y LAS TURBAS EMPLEADAS PARA LA FORMACIÓN DE LOS SUSTRATOS $(\%$ de volumen)

\begin{tabular}{lcccc}
\hline $\begin{array}{l}\text { Sustratos y mezclas } \\
\text { con pollinazas }\end{array}$ & $\begin{array}{c}\text { Pollo engorde } \\
\text { paja }(\%)\end{array}$ & $\begin{array}{c}\text { Pollo engorde } \\
\text { serrín }(\%)\end{array}$ & $\begin{array}{c}\text { Turba negra } \\
(\%)\end{array}$ & $\begin{array}{c}\text { Turba rubia } \\
(\%)\end{array}$ \\
\hline 100TN & 0 & 0 & 100 & 0 \\
$90 \mathrm{TN}+10 \mathrm{PEP}$ & 10 & 0 & 90 & 0 \\
90TN+ 10PES & 0 & 10 & 90 & 0 \\
100TR & 0 & 0 & 0 & 100 \\
90TR+ 10PEP & 10 & 0 & 0 & 90 \\
90TR+ 10PES & 0 & 10 & 0 & 90 \\
\hline
\end{tabular}

$\mathrm{TN}=$ Turba negra, $\mathrm{TR}=$ Turba rubia, $\mathrm{PES}=$ Pollo de engorde-serrín, $\mathrm{PEP}=$ Pollo de engorde-paja 
CUADRO II. CARACTERIZACIÓN QUÍMICA DE LAS POLLINAZAS Y LAS TURBAS EMPLEADAS PARA LA FORMACIÓN DE LOS SUSTRATOS. DESVIACIÓN ESTÁNDAR ENTRE PARÉNTESIS

\begin{tabular}{lccrr}
\hline & Pollinaza paja & Pollinaza serrín & Turba negra & Turba rubia \\
\hline Nitrógeno Kjeldahl (\%) & $4.57(0.86)$ & $3.46(0.03)$ & $0.98(0.33)$ & $1.14(0.34)$ \\
$\mathrm{NH}^{+}{ }_{4}-\mathrm{N}(\mathrm{mg} / \mathrm{kg})$ & $8261.47(381.62)$ & $6125.01(696.26)$ & $58.93(1.39)$ & $61.26(1.38)$ \\
$\mathrm{NO}^{-}{ }_{3}-\mathrm{N}(\mathrm{mg} / \mathrm{kg})$ & $235.58(63.90)$ & $203.50(35.23)$ & $1602.82(1.05)$ & $1311.58(1.70)$ \\
$\mathrm{pH}\left(\mathrm{H}_{2} \mathrm{O}\right)$ & $7.66(0.23)$ & $8.47(0.10)$ & $6.17(0.12)$ & $3.52(0.07)$ \\
$\mathrm{C} . \mathrm{E}(\mathrm{dS} / \mathrm{m})$ & $11.21(3.85)$ & $8.59(1.11)$ & $0.95(0.24)$ & $0.37(0.08)$ \\
$\mathrm{CO}(\%)$ & $35.72(2.95)$ & $35.67(1.07)$ & $28.16(2.57)$ & $22.75(2.29)$ \\
$\mathrm{C} / \mathrm{N}$ & 7.82 & 10.31 & 28.73 & 19.96 \\
Total P $(\%)$ & $0.95(0.32)$ & $0.93(0.41)$ & $0.93(0.41)$ & $0.95(0.31)$ \\
Total K $(\%)$ & $1.91(0.41)$ & $2.01(1.23)$ & $1.96(1.21)$ & $2.17(1.22)$ \\
\hline
\end{tabular}

CUADRO III. pH Y CONDUCTIVIDAD ELÉCTRICA DE LOS DIFERENTES SUSTRATOS FORMULADOS (\% DE VOLUMEN). DESVIACIÓN ESTÁNDAR ENTRE PARÉNTESIS

\begin{tabular}{lcc}
\hline $\begin{array}{l}\text { Sustratos y mezclas } \\
\text { con pollinazas }\end{array}$ & $\begin{array}{c}\mathrm{pH} \\
1: 5 \mathrm{H}_{2} \mathrm{O}\end{array}$ & $\begin{array}{c}\text { Conductividad eléctrica } \\
1: 5 \mathrm{H}_{2} \mathrm{O} \\
\mathrm{dS} / \mathrm{m}\end{array}$ \\
\hline $100 \mathrm{TN}$ & $6.17(0.12)$ & $0.95(0.24)$ \\
$90 \mathrm{TN}+10 \mathrm{PEP}$ & $5.68(0.10)$ & $3.91(1.00)$ \\
$90 \mathrm{TN}+10 \mathrm{PES}$ & $5.73(0.10)$ & $4.03(1.00)$ \\
$100 \mathrm{TR}$ & $3.52(0.07)$ & $0.37(0.08)$ \\
$90 \mathrm{TR}+10 \mathrm{PEP}$ & $4.62(0.08)$ & $1.18(0.24)$ \\
$90 \mathrm{TR}+10 \mathrm{PES}$ & $4.51(0.08)$ & $1.67(0.24)$ \\
\hline
\end{tabular}

$\mathrm{TN}=$ Turba negra, $\mathrm{TR}=$ Turba rubia, $\mathrm{PES}=$ Pollo de engordeserrín, $\mathrm{PEP}=$ Pollo de engorde-paja

- Densidad aparente (Da): es la masa por unidad de volumen, incluido el volumen de poros entre las partículas $\left(\mathrm{g} / \mathrm{cm}^{3}\right)$.

- Porosidad total (Po): es el porcentaje del volumen de poros llenos de aire y de agua, y por lo tanto no ocupado por las partículas, en relación al volumen total (\%).

- Humedad en volumen (Hv): Es el porcentaje de agua en volumen (\%). Se calcula, $\mathrm{Hv}=\mathrm{Da} \times \mathrm{H}$

- Capacidad de aireación (Ca): comprende el volumen de aire (Va) del sustrato sometido a una tensión de $10 \mathrm{~cm}$ de columna de agua. Se obtiene, $\mathrm{Va}=$ Po Hv (a $10 \mathrm{~cm})$.

- Agua fácilmente utilizable (AFU): cantidad de agua que se libera al aplicar una tensión al sustrato de entre 10 y $50 \mathrm{~cm}$ de columna de agua (\%). Se calcula, Hv10 Hv 50.

- Capacidad compensadora de agua (CCA): cantidad de agua que se libera al aplicar una tensión al sustrato de entre 50 y $100 \mathrm{~cm}$ de columna de agua (\%). Se calcula, Hv 50 Hv 100.
- Agua difícilmente utilizable (ADU): cantidad de agua que queda retenida en el sustrato después de aplicar una tensión de $100 \mathrm{~cm}$ de columna de agua (\%). Se calcula, Po CCA y AFU Va10.

- Valor R: se define como la succión a la que el volumen de aire es igual al volumen de agua (Felipó et al. 1979), se calcula como el punto de corte de la curva de humedad volumétrica con la del volumen de aire en las gráficas de distribución de la porosidad.

\section{Análisis estadístico}

Para determinar las diferencias significativas entre sustratos se realizó una prueba de Duncan $(\mathrm{P} \leq 0.05)$, con el programa estadístico Statgraphics Plus 5.1 (SAS 2011).

\section{RESULTADOS Y DISCUSIÓN}

En el cuadro II se presentan las propiedades químicas iniciales de las pollinazas y los sustratos estudiados. Se puede observar que el contenido de carbono orgánico fue similar cuando se comparan las dos pollinazas y las dos turbas, pero se encontraron diferencias al contrastar las pollinazas con las turbas. La relación $\mathrm{C} / \mathrm{N}$ se redujo de manera importante para las pollinazas estudiadas, debido a su alto contenido en nitrógeno total.

En el cuadro III que muestra el $\mathrm{pH}$ y la $\mathrm{CE}$ de los sustratos (TN y TR) y pollinazas (PEP y PES), se puede observar un $\mathrm{pH}$ muy ácido en el $100 \%$ de TR, por lo que resulta no adecuada para el cultivo del crisantemo. Con la adición del $10 \%$ PEP o PES el pH aumenta, lo que a su vez incrementa la salinidad, aunque dentro de los parámetros indicados para el cultivo del crisantemo (Miralles de Imperial et al. 2008, Alonso et al. 2012). 
La utilización de las mezclas de TN con PES y PEP, por su alto valor de CE, estaría condicionada al estudio de sus propiedades físicas e hidrofísicas.

Kreij y Van Leeuwen (2001) consideraron que los valores de $\mathrm{pH}$ óptimos para el cultivo de crisantemo, están entre 4.8 y 5.9. Mientras que Benito et al. (2002) y Kämpf et al. (2006), señalan que está entre 6 y 7. Por otro lado, Jiménez y Caballero (1990) afirman que el $\mathrm{pH}$ óptimo de la mezcla del sustrato para este cultivo es de 6 .

Valores de conductividad entre 2 y $3.5 \mathrm{dS} / \mathrm{m}$ se consideran adecuados para la mayoría de los cultivos, entre 3.5 y 5 se consideran ligeramente altos (para plantas resistentes a la salinidad) y entre 0.75 y 1.99 se consideran bajos, adecuados para semilleros y plántulas (Guerrero y Masaguer 1997, Fascella 2015).

Alonso et al. (2012) estudiaron la influencia del $\mathrm{pH}$ y la $\mathrm{CE}$ de diferentes mezclas de sustratos y concluyeron que la supervivencia del cultivo no quedaba afectada en ninguna de sus mezclas, pero sí en su crecimiento, en el que se presentó una disminución con la mezcla de turba rubia (TR+PEP y TR+PES).

Asimismo, es muy importante conocer las propiedades físicas de los sustratos antes de su utilización, debido a que una vez que se encuentren en el contenedor y la planta esté creciendo en él, no es posible modificar sus características físicas básicas (Abad y Noguera 1998). En particular, la Da tiene un papel relevante, ya que los sustratos y contenedores se transportan durante su manejo y manipulación.

En el cuadro IV se muestran los distintos parámetros de las determinaciones físicas e hidrofísicas de los sustratos estudiados.

La humedad natural (producto fresco) de todos los tratamientos está por encima del $50 \%$, valor por debajo del cual no es recomendable que se encuentre un material orgánico, ya que si fuera necesario humedecerlo resultaría muy laborioso, requeriría de tiempo y se presentarían pérdidas económicas (Masaguer et al. 1997).

Los sustratos y las mezclas con pollinaza presentaron una elevada porosidad, superior al $90 \%$, aunque no hubo diferencias significativas entre ellos $(\mathrm{P}>0.05)$, lo cual permite suponer que existió una aireación suficiente, incluso compatible con una elevada retención de humedad (Cruz et al. 2010).

Cuando se estudió la curva de humedad a bajas succiones se pudo comprobar que la capacidad de aireación $(\mathrm{Ca})$, es superior a $20 \%$ y por tanto adecuada, excepto en el sustrato obtenido de la mezcla con $90 \mathrm{TN}+10 \mathrm{PES}$ que es de $16 \%(\mathrm{P}<0.05)$. Este es un factor importante para evitar la asfixia radicular que puedan tener algunos cultivos de especies más sensibles (Amorin et al. 2012).

El AFU, es menor al $20 \%$ por lo que se encuentra por debajo de los valores entre 20 y 30 , recomendados por Abad y Noguera (1998). Solamente las mezclas de $90 \mathrm{TN}+10$ PES alcanzaron el valor óptimo $(\mathrm{P}<0.05)$.

En general, en todos los sustratos ensayados el AFU es escasa. Sin embargo, hay que destacar la mejora que se produce con las mezclas PEP, puesto que se reduce el ADU.

Este efecto benéfico de las propiedades físicas con la incorporación del PEP también se aprecia al estudiar las curvas de retención de humedad (Figs. 1 a 6 ).

Para los sustratos comerciales (TN y TR) las curvas presentan un comportamiento general correcto, con un equilibrio aire-agua $(\mathrm{R})$ próximo a 30 , lo que indica que el equilibrio se produce a una tensión de $30 \mathrm{~cm}$ de columna de agua, muy habitual en contene-

CUADRO IV. PROPIEDADES FÍSICAS E HIDROFÍSICAS DE LOS SUSTRATOS FORMULADOS

\begin{tabular}{lcccccccc}
\hline $\begin{array}{l}\text { Sustratos y mezclas } \\
\text { con pollinazas }\end{array}$ & $\begin{array}{c}\mathrm{H} \\
(\%)\end{array}$ & $\begin{array}{c}\mathrm{Da} \\
\left(\mathrm{g} / \mathrm{cm}^{3}\right)\end{array}$ & $\begin{array}{c}\text { Po } \\
(\%)\end{array}$ & $\begin{array}{c}\mathrm{Ca} \\
(\%)\end{array}$ & $\begin{array}{c}\text { AFU } \\
(\%)\end{array}$ & $\begin{array}{c}\text { CCA } \\
(\%)\end{array}$ & $\begin{array}{c}\text { ADU } \\
(\%)\end{array}$ & $\begin{array}{c}\mathrm{R} \\
(\mathrm{cm})\end{array}$ \\
\hline $100 \mathrm{TN}$ & $67.72 \mathrm{~d}(0.86)$ & $0.12 \mathrm{a}(0.08)$ & $92 \mathrm{a}(0.31)$ & $28 \mathrm{c}(0.21)$ & $15 \mathrm{a}(0.15)$ & $4 \mathrm{c}(0.07)$ & $46 \mathrm{c}(0.57)$ & $30 \mathrm{~b}(0.35)$ \\
$90 \mathrm{TN}+10 \mathrm{PEP}$ & $57.02 \mathrm{~b}(0.35)$ & $0.12 \mathrm{a}(0.08)$ & $92 \mathrm{a}(0.31)$ & $37 \mathrm{~d}(0.21)$ & $13 \mathrm{a}(0.18)$ & $4 \mathrm{c}(0.10)$ & $39 \mathrm{a}(0.35)$ & $11 \mathrm{a}(0.24)$ \\
$90 \mathrm{TN}+10 \mathrm{PES}$ & $52.14 \mathrm{a}(0.27)$ & $0.14 \mathrm{~b}(0.08)$ & $91 \mathrm{a}(0.31)$ & $16 \mathrm{a}(0.24)$ & $20 \mathrm{~b}(0.15)$ & $4 \mathrm{c}(0.10)$ & $51 \mathrm{~d}(0.50)$ & $55 \mathrm{~d}(0.30)$ \\
$100 \mathrm{TR}$ & $67.90 \mathrm{~d}(0.86)$ & $0.13 \mathrm{~b}(0.08)$ & $91 \mathrm{a}(0.31)$ & $24 \mathrm{~b}(0.24)$ & $15 \mathrm{a}(0.15)$ & $1 \mathrm{a}(0.05)$ & $52 \mathrm{~d}(0.50)$ & $39 \mathrm{c}(0.35)$ \\
$90 \mathrm{TR}+10 \mathrm{PEP}$ & $68.62 \mathrm{~d}(0.90)$ & $0.13 \mathrm{~b}(0.08)$ & $92 \mathrm{a}(0.31)$ & $28 \mathrm{c}(0.25)$ & $19 \mathrm{~b}(0.10)$ & $3 \mathrm{~b}(0.05)$ & $43 \mathrm{~b}(0.57)$ & $28 \mathrm{~b}(0.30)$ \\
90TR+10PES & $62.44 \mathrm{c}(0.80)$ & $0.14 \mathrm{~b}(0.08)$ & $91 \mathrm{a}(0.31)$ & $22 \mathrm{~b}(0.24)$ & $15 \mathrm{a}(0.10)$ & $5 \mathrm{~d}(0.07)$ & $48 \mathrm{c}(0.50)$ & $50 \mathrm{~d}(0.35)$ \\
Nivel óptimo* & - & $<0.40$ & $>80$ & $20-30$ & $20-30$ & $4-10$ & $25-31$ & $10-30$ \\
\hline
\end{tabular}

*Abad y Noguera 1998

$\mathrm{TN}=$ Turba negra, TR $=$ Turba rubia, PES $=$ Pollo de engorde-serrín, PEP $=$ Pollo de engorde-paja, $\mathrm{H}=$ humedad, Da $=$ densidad aparente, $\mathrm{Po}=$ porosidad, $\mathrm{Ca}$ = capacidad de aireación, $\mathrm{AFU}$ = agua fácilmente utilizable, $\mathrm{CCA}=$ capacidad compensadora de agua, $\mathrm{ADU}=$ agua difícilmente utilizable, $\mathrm{R}=$ parámetro $\mathrm{R}$. Letras distintas en la misma columna indican que existe diferencia significativa $(\mathrm{P} \leq 0.05)$. Desviación estándar entre paréntesis 


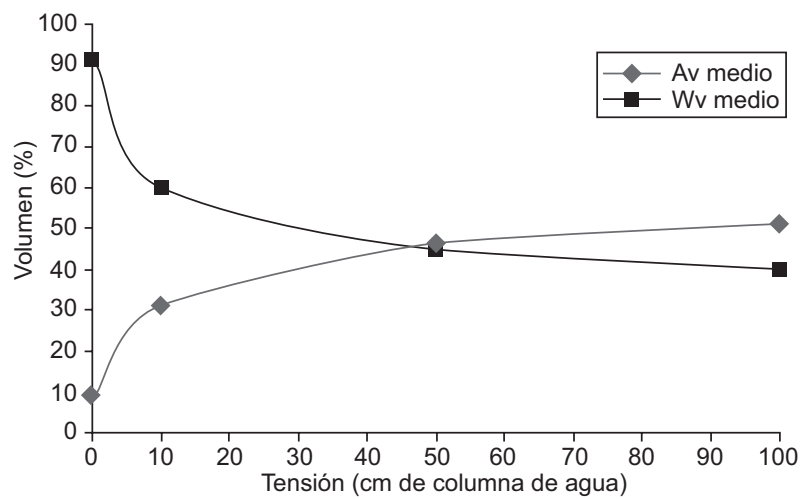

Fig. 1. Curva característica de humedad del sustrato turba negra. Volumen medio de aire (Av) y volumen medio de agua $(\mathrm{Wv})$

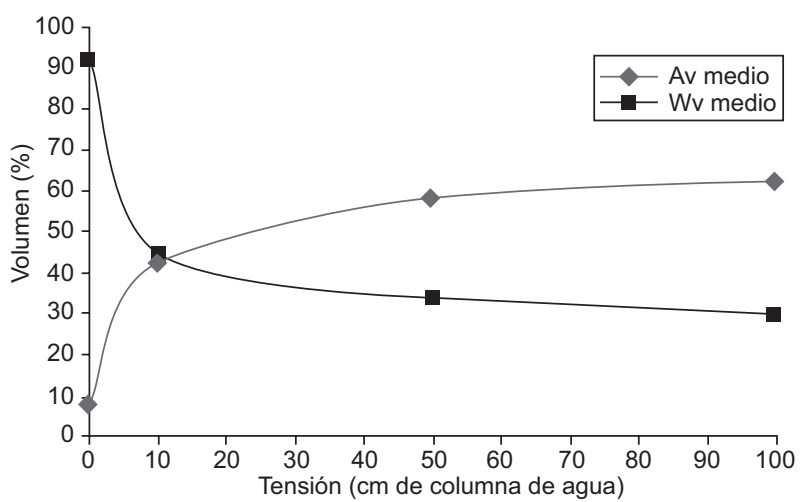

Fig.2. Curva característica de humedad del sustrato $90 \%$ turba negra $+10 \%$ pollo de engorde paja. Volumen medio de aire $(\mathrm{Av})$ y volumen medio de agua (Wv)

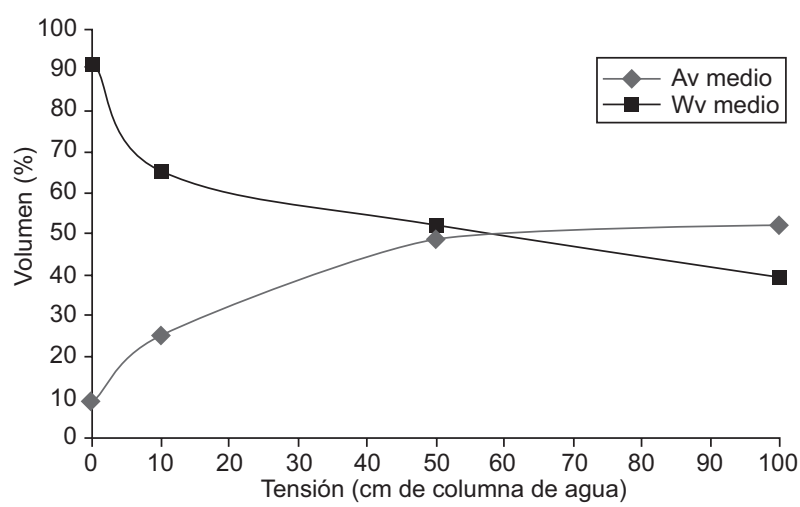

Fig.3. Curva característica de humedad del sustrato $90 \%$ turba negra $+10 \%$ pollo de engorde serrín. Volumen medio de aire $(\mathrm{Av})$ y volumen medio de agua (Wv)

dores con riego adecuado. Se observa cómo la incorporación de PEP reduce esta tensión hasta 11 en TN + PEP y 28 en TR + PEP, lo que se encuentra en los valores recomendados (10-30) (Aceved y Pire 2007).

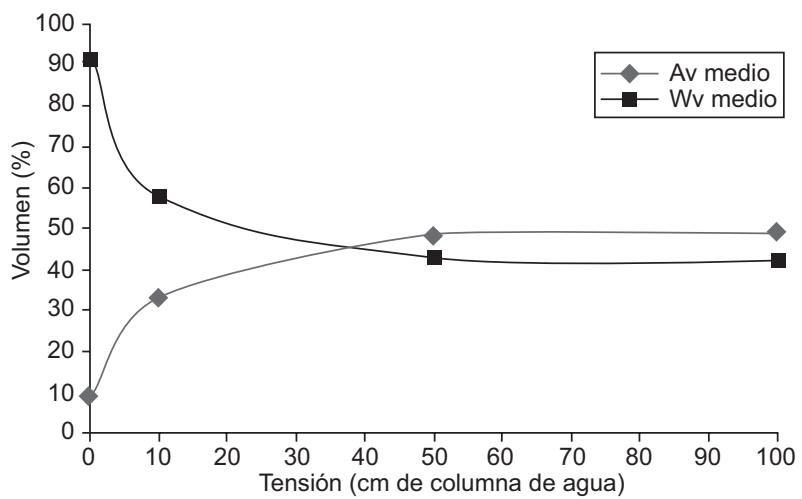

Fig.4. Curva característica de humedad del sustrato turba rubia. Volumen medio de aire (Av) y volumen medio de agua (Wv)

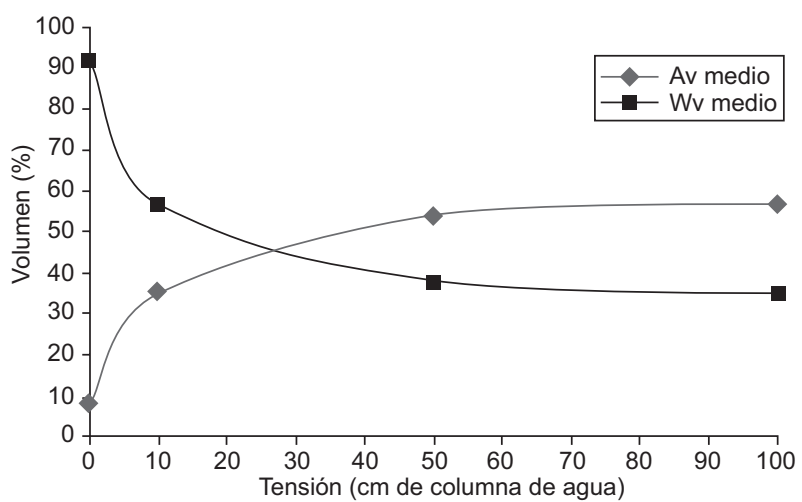

Fig.5. Curva característica de humedad del sustrato $90 \%$ turba rubia $+10 \%$ pollo de engorde paja. Volumen medio de aire (Av) y volumen medio de agua (Wv)

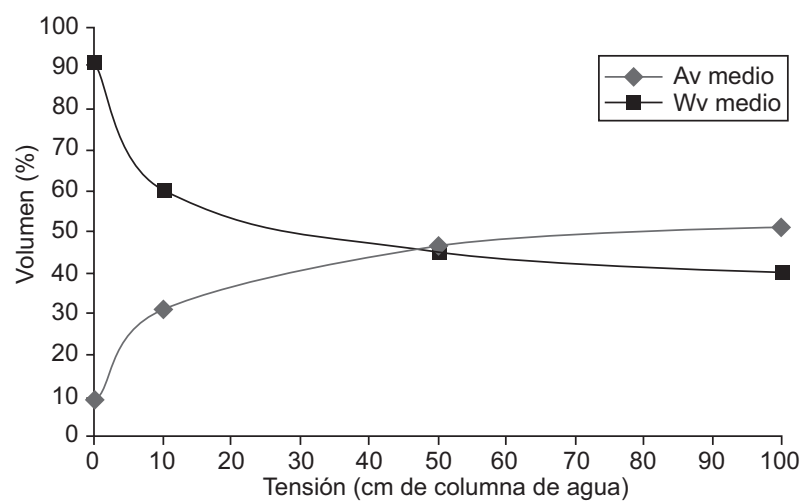

Fig.6. Curva característica de humedad del sustrato $90 \%$ turba rubia $+10 \%$ pollo de engorde serrín. Volumen medio de aire (Av) y volumen medio de agua (Wv)

Por otra parte, la incorporación de pollinaza PES en la mezcla confiere mayor retención de humedad e incrementa R hasta valores de $50-55 \mathrm{~cm}$. Sin embargo, no existen diferencias significativas cuando 
se mezcla esta pollinaza con turba negra $(90 \mathrm{TN}+$ $10 \mathrm{PES}$ ) o con turba rubia (90 TR+ $10 \mathrm{PES})$. Estos resultados no implican que se reduzca el valor del material, simplemente que aumenta su capacidad de retener agua y así se podrían espaciar más los riegos.

La valorización económica de los residuos orgánicos avícolas puede suponer una reducción interesante para la fabricación de sustratos de cultivo. Se puede estimar que añadiendo un $10 \%$ de residuo a la mezcla de sustrato supondría un ahorro global de 3 millones de euros (U.S. Geological Survey 2012).

Al balance económico positivo se debe añadir el valor ambiental que supone la reducción en la extracción de turba en ecosistemas frágiles, que son una importante reserva de carbono, por lo que se mitigaría directamente el cambio climático.

\section{CONCLUSIONES}

Las mezclas de turba negra con PES y PEP pueden incrmentar la CE. Esto puede subsanarse con un riego abundante al principio del cultivo y con la PEP que mejora las propiedades físicas e hidrofísicas.

Para la TR el incremento de la CE fue menor y el $\mathrm{pH}$, aunque es bajo, no representa problema alguno, ya que de cualquier manera se mejoran las propiedades físicas con la incorporación de la PEP a la mezcla.

Se puede asegurar que las mezclas con los dos subproductos estudiados son válidas para ser utilizadas como sustrato de cultivo, debido a la mejora de las propiedades físicas del mismo. Sólo habría que considerar las propiedades físicas e hidrofísicas (H, Da, Po, Ca, AFU, CCA, ADF y R) y establecer el manejo adecuado, como el control de riegos para evitar la salinidad, en particular con la TN.

\section{AGRADECIMIENTOS}

Este estudio se realizó gracias a los proyectos RTA2005-00120-CO2-01 y RTA2009-00074-00-00 financiados por el Instituto Nacional de Investigación y Tecnología Agraria y Alimentaria (INIA-FEDER). Los autores agradecen a Jesús García, María Isabel González y Ángela García por su colaboración en las tareas de campo y de laboratorio.

\section{REFERENCIAS}

Abad M. y Noguera P. (1998). Los sustratos para el cultivo sin suelo y fertirrigación. Mundi- Prensa, España, 250 pp.
Aceved I. C. y Pire R. (2007). Caracterización de sustratos agrícolas enmendados com lombricompost. Rev. Unellez de Ciencia y Tecnología 254, 1-9.

AENOR (2012). Norma UNE-EM 13041:2012. Mejoradores de suelo y sustratos de cultivo. Determinación de las propiedades físicas. Densidad aparente seca, volumen de aire, volumen de agua, valor de contracción y porosidad total. Asociación Española de Normalización y Certificación. 15 abril 2012.

Alonso F., Miralles de Imperial R., Martín J. V., Rodríguez C. y Delgado M. (2012). Responses of chrysanthemum plant pot growth to commercial plant substrate with broiler manure. Rev. Int. Contam. Ambie. 27, 171-179.

Amorin A., Ribeiro S., Marques de Faria R., Previdelli M.A. y Garofallo R. (2012). Effect of diferente substrates on composting of poultry litter. R. Bras. Zootec. 41, 1764-1768.

DOI: $10.1590 / \mathrm{S} 1516-35982012000700028$

Benito M., Masaguer A., De Antonio R. y Moliner A. (2002). Potential use of pruning waste compost as a growing media for ornamental plants. Compost Sci. Util. 30, 1-8.

Cruz E., Sandoval M., Volke V., Ordaz V. y Sánchez J. (2010). Generación de mezclas de sustratos mediante un programa de optimización utilizando variables físicas y químicas. Terra Latinoamericana 28, 219-229.

De Boodt M., Verdonck O. y Cappaert L. (1974). Method for measuring the water release curve of organic substrates. Acta Hort. 37, 2054-2062.

DOI: 10.17660/ActaHortic.1974.37.20

Delgado M., Miralles de Imperial R. y Martín J. V. (2007). Evaluación de granjas intensivas de producción avícola. Rev. EIDENAR 6, 33-39.

Delgado M., Martín J.V., Miralles de Imperial R., León C. y García M. C. (2010). Phytotoxicity of uncomposted and composted poultry manure. Afr. J. Plant Sci. 4, 154-162.

Felipó M.T., Verdonck L., Cappaert L. y De Boodt, M. (1979). Estudio de las propiedades físicas de los sustratos hortícolas. Anal. Edaf. Agrobiol. 38, 603-611.

García M. C., León C., Delgado M., Miralles de Imperial R. y Martín J. V. (2007). Caracterización, manejo y diferencias entre los residuos procedentes de granjas ponedoras y pollos broiler en explotaciones de Castilla y León. Tecnoganadería 5, 16-18.

Fascella G. (2015). Growing substrates alternative to peat of ormanamental plants. Chapter 3. En: Soilless culture - Use of substrates for the production of quality horticultural crops (Md. Asaduzzaman, Ed.). Asadurraman. Rijeka, Croacia, pp. 47-68.

DOI: $10.5772 / 59596$

Guerrero F. y Masaguer A. (1997). Los sustratos en los semilleros hortícolas. Vida Rural 47, 52-56. 
IGME (2010). Memoria de atividades del Instituto Geológico y Minero de España. Instituto Geológico Minero (IGM), Madrid, España, 95 pp.

Jiménez R. y Caballero M. (1990). El cultivo industrial de plantas en maceta. Ediciones de Horticultura. Madrid, España, 664 pp.

Kämpf A.N., Jun R. y Vital P.T. (2006). Floricultura. Técnicas de preparo de substratos. LK editora. Brasilia, Brasil, 132 pp.

Kreij C. y Van Leeuwen G. J. (2001). Growth of pot plants in treated coir dust as compared to peat. Commun. Soil. Sci. Plant. Anal. 32, 2255-2265.

DOI: $10.1081 /$ CSS-120000281

López M.C., Ruiz J. y Masaguer A. (2006). Producción de planta ornamental en contenedor con sustratos alternativos a la turba. Ensayos en la Comunidad de Madrid. Colección de investigación del Instituto Madrileño de Desarrollo Regional Agrário (IMIDRA). Madrid, España, 176 pp.

MAPA (1994). Métodos oficiales de análisis. Tomo III. Ministerio de Agricultura, Pesca y Alimentación. Madrid, España, 662 pp.
Masaguer A., Moliner A. y Guerrero F. (1997). Evaluación agronómica de diferentes cortezas de pino para la producción de Pinus pinea en contenedor. Actas de Horticultura 17, 82-88.

Miralles de Imperial R., Martín J. V., García. M. C., León. C. y Delgado M. M. (2008). Producción de crisantemo en macetas, comportamiento en sustratos con turbas y mezclas con gallinazas. Plantflor Cultivo y Comercio $130,101-103$.

SAS (2011). SAS/STAT 9.3 User's guide. Statical Analysis Systems, Institute Inc. Cary, EUA, 178 pp.

Schellekens J., Buurman P. Fraga I. y Martínez-Cortizas A. (2011). Holocene vegetation and hydrologic changes inferred fom molecular vegetation markers in peat, Penido Vello (Spain). Palaeogeography 299, 56-69. DOI: 10.1016/j.palaeo.2010.10.034

U.S. Geological Survey (2012). Mineral commodity sumaries 2012: U.S. Geological Survey, EUA, 198 pp. 